\title{
Induction, Rational Acceptance, and Minimally Inconsistent Sets
}

1. Introduction. The purpose of this paper is to present a theory of inductive inference and rational acceptance in scientific inquiry. A concept of relevant deduction is defined in which the truth of each and every premise of a deductive argument is essential to establishing the truth of the conclusion by deduction from the premises. This definition is based on the completely abstract notion of a minimally inconsistent set of statements. In terms of this same abstract logical concept and the relation of probability, we shall define a concept of inductive inference that is a principle of rationality. This concept of inductive inference is shown to form the basis of a principle of acceptance in which two important epistemic utilities are maximized.

2. Conditions of Relevant Deduction. Suppose we are engaged in reasoning concerning some scientific conclusion that is neither contradictory nor logically true. If we intend to establish the truth of the conclusion by deduction from the premises, then it is important that several conditions be met to ensure that the deductive argument is relevant to that end. First the premises of such a relevant deductive argument, RD, must be logically consistent:

CRl. The set of premises $P 1, P 2, \ldots$, and $P_{\mathrm{n}}$ of $R D$ is logically consistent.

The need for imposing (CR1) as a condition of relevance arises because an argument with an inconsistent set of premises is valid no matter what conclusion the argument has. An inconsistent set of premises is quite irrelevant to establishing the truth of a conclusion deduced from them. Some philosophers and logicians have maintained that every statement is relevant to itself and hence that a contradiction is relevant to itself. But this concept of relevance is a different one from that under 


\section{Keith Lehrer}

consideration here. ${ }^{1}$ I am concerned with a concept of relevance such that the premises of an argument are relevant to establishing the truth of the conclusion and nothing is relevant to establishing the truth of a contradiction.

The second condition of relevance is that all the premises be essential to the deduction of the conclusion from the premises. An argument is only as relevant as its premises, and thus if an argument contains an irrelevant or inessential premise it is not completely relevant. We may formulate this condition as follows:

CR2. The set of premises $P 1, P 2, \ldots, P \mathbf{n}$ of $R D$ is nonempty and such that no proper subset of the set is sufficient for the deduction of the conclusion $C$ of $R D$.

This condition is required to ensure that the argument is relevant, but it is also useful for the clarification, defense, and criticism of arguments in a scientific context. Suppose that a deductive argument is offered in defense of a scientific conclusion when the argument does not satisfy condition (CR2). In that case, the investigator may be misled into thinking that evidential support is required in defense of his conclusion because it is needed for the defense of some inessential premise. When such a premise is inessential for the deduction of the conclusion, the evidence supporting it may be otiose. Similarly, if an argument does not satisfy (CR2), then criticism of the argument directed against some premise of the argument may be fruitless because the premise is inessential to the deduction of the conclusion. Thus, if a deductive argument intended to establish the truth of a scientific conclusion satisfies (CR2), this will help to avoid useless evidence gathering and equally fruitless criticism.

The characterization of relevance contained in condition (CR2) logically entails the result that no argument having a tautological conclusion (and one or more premises) is a relevant deductive argument. This is a consequence of the feature of systems of natural deduction in which any tautology may be derived by conditional proof from the null set of premises. Thus, given (CR2), it follows that any argument having a tautological conclusion and one or more premises will fail to be relevant

${ }^{1}$ See A. R. Anderson and N. D. Belnap, "The Pure Calculus of Entailment," Journal of Symbolic Logic, 27 (1962), 19-52, esp. 20, 36, 39, and 42. Various systems by other authors are discussed which postulate that $P$ is relevantly deducible from $P$ even when $P$ is contradictory. 
because the null set of premises is sufficient for the deduction of the conclusion. Moreover, no argument with a tautological premise will be relevant either. Again given a system of natural deduction, any set of premises containing a tautology is such that the proper subset of premises containing only those premises which are not tautological will suffice for the deduction of the conclusion. Therefore, an argument that is relevant in the sense that the truth of each and every premise is essential to establishing the truth of the conclusion by deduction from premises will have neither tautological premises nor a tautological conclusion. This follows from (CR2). It follows from (CR1) that no premise of a relevant deductive argument may be contradictory, and obviously no contradiction can be the conclusion of such an argument.

The preceding results indicate that a deductive argument is relevant in the sense under consideration only if the premises and the conclusion of the argument are all logically contingent, that is, neither contradictory nor tautological. Though this condition is not independent of the two previous conditions, we shall add it as a third condition.

$C R 3$. The premises $P 1, P 2, \ldots, P n$ of $R D$ as well as the conclusion $C$ of $R D$ are neither contradictions nor tautologies.

This condition ensures that relevant deductive arguments are ones in which the truth of contingent premises, and only such, is essential to establishing the truth of a contingent conclusion by deducing the latter from the former.

It might seem to be an objection to the preceding characterization of relevance that an argument can be shown to be irrelevant in a scientific context by virtue of the fact that the conclusion of the argument can be established by deduction from totally different premises. However, it is not this sense of relevance I am attempting to explicate. I am only attempting to explicate that sense in which an argument is relevant because the truth of each and every premise of the argument is essential to establishing the truth of the conclusion by deduction from those premises. Thus, I am only concerned with the relevance of the premises within the argument and not with the more general question of whether the argument is required at all.

3. Analysis of Relevant Deduction. Having laid down three conditions of relevant deductive argument, I shall now offer an analysis of the concept of relevance under consideration. First, let us recall that a valid 


\section{Keith Lehrer}

deductive argument is such that the set of statements consisting of the premises of the argument and the denial of the conclusion (or any truth functional equivalent) is logically inconsistent, that is, one may deduce a contradiction from the set of statements. A valid deductive argument that is also relevant is one such that the set of statements having as members the premises and the denial of the conclusion (or any truth functional equivalent) is not only logically inconsistent but minimally inconsistent. A set of statements is minimally inconsistent if and only if the set is logically inconsistent and such that no proper subset of the set is logically inconsistent. In short, a minimally inconsistent set is one such that all the statements of the set are required to make it inconsistent.

The preceding considerations yield the following analysis of a relevant deductive argument:

ARl. An argument $R D$ is a relevant deductive argument if and only if $R D$ contains a nonempty set of premises $P 1, P 2$, ..., Pn and a conclusion $C$ such that a set of statements consisting of just $P 1, P 2, \ldots, P n$, and $\sim C$ (or any truth functional equivalent of $\sim C$ ) is a minimally inconsistent set. A set of statements is a minimally inconsistent set if and only if the set of statements is logically inconsistent and such that every proper subset of the set is logically consistent.

It follows from (AR1) that conditions (CR1), (CR2), and (CR3) are all met. The proof that (CR1) is met is that if the premises of the argument $R D$ were inconsistent, then the set of premises would be an inconsistent proper subset of the set of statements consisting of the premises and the denial of the conclusion (or some truth functional equivalent). The proof that (CR2) is met is that if the set of premises of the argument $R D$ were such that a proper subset of the set was sufficient for deduction of the conclusion, then the set of statements consisting of that proper subset of the premises and the denial of the conclusion would be an inconsistent proper subset of the set of statements consisting of all the premises of $R D$ and the denial of the conclusion. The proof that (CR3) is met is that any two-membered minimally inconsistent set is such that neither member may be a contradiction or a tautology. For if one statement were a tautology, the other would have to be a contradiction to make the set inconsistent, in 
which case the proper subset containing just the contradiction would be an inconsistent set. If one statement of a two-membered set is a contradiction, then the proper subset of the set containing that contradiction as an only member would be an inconsistent set. Thus conditions (CR1), (CR2), and (CR3) are logical consequences of (ARl).

The preceding discussion characterized and analyzed a concept of a relevant deductive argument. The concept is of some interest simply because any argument in which the truth of a conclusion is established by deduction from premises must always be such that either it is a relevant deductive argument or else some relevant deductive argument could be substituted for it, thereby clarifying which premises of the original argument are essential. By means of such clarification we may avoid ineffective criticism and superfluous defense.

4. Induction. The preceding discussion was concerned with relevant deductive arguments in which the truth of each and every premise is required to establish the truth of the conclusion by deduction from the premises. The question to which we shall now turn is that of investigating the role of such arguments in scientific inquiry. A relevant deductive argument may fail to establish the conclusion because the epistemic worth of the argument depends on the value of the premises. If the premises are without merit and would not be accepted in scientific inquiry, then the argument is ineffective for the purpose of gaining acceptance for the conclusion. Consequently, any adequate account of justificatory reasoning must be based on an explication of the rational acceptance of statements as premises.

Philosophers have notoriously disagreed about the status of such premises. Some have argued that premises should be confined to observation reports and others have argued to the contrary that this restriction is unrealistic epistemologically and/or semantically. It is unrealistic epistemologically if it would prohibit us from accepting well-established scientific theories, and it is unrealistic semantically if the distinction between theoretical terms and observation terms is untenable. I shall not here attempt to resolve such disputes. Instead, I shall leave open the question of the exact character of initial scientific premises, if there are such premises, and consider the epistemological situation we confront in any given stage in the history of scientific inquiry. Ultimately, my reason for doing so is that I believe scientific inquiry can proceed quite nicely without any permanent foundation of statements that 


\section{Keith Lehrer}

justify other statements without being so justified themselves. However, even if I am quite mistaken in this assumption, there is some methodological justification for putting the question of ultimate premises to rest and attempting to explicate the character of scientific inference as it proceeds against the background of premises accepted as belonging to the body of scientific knowledge. Thus, I shall assume that we have such a background of accepted statements and examine the character of inductive reasoning.

Though some reasoning may be explicated as reasoning from accepted premises to conclusions by means of relevant deductive argument, other reasoning may not be so explicated and this I shall refer to as inductive reasoning. The aims of inductive reasoning have proven to be controversial. Since an inductive argument is not necessarily truth preserving, we cannot affirm that our only objective in inductive argument is to arrive at the truth. On the contrary, if all we cared about was that the conclusion of our argument be true, then we would not be justified in venturing beyond the deductive consequences of our premises. For even if our premises are true, to reason inductively is to risk error and for such a risk there must be some redeeming benefit. ${ }^{2}$ A number of such benefits have been proposed. One is that of explanation, that it is an aim of scientific inquiry to arrive at conclusions that explain and that are explained. ${ }^{3}$ Another aim is to arrive at conclusions that give us additional informational content. ${ }^{4}$ When we risk error in inductive reasoning, the risk is justified by the fact that the conclusions at which we arrive will, if true, add to our information and facilitate explanation. Of course, the goal of arriving at true conclusions is not to be eschewed, but neither should it exclude other cognitive interests.

This approach to inductive reasoning is the one that I shall adopt.

${ }^{2}$ Cf. Isaac Levi, Gambling with Truth (New York: Knopf, 1967), pp. 119-28.

${ }^{3}$ This goal has been stressed by many writers, especially Karl Popper, W. V. O. Quine, W. H. Sellars, Gilbert Harman, and Paul Ziff. In an earlier paper, Keith Lehrer, "Justification, Explanation, and Induction," in M. Swain, ed., Induction, Acceptance and Rational Belief (Dordrecht: Reidel, 1970), pp. 119-20, I present a rule designed to achieve this objective.

'See Levi, Gambling with Truth, pp. 57-90, Karl Popper, The Logic of Scientific Discovery (London: Hutchinson, 1959), pp. 119-21, Jaakko Hintikka and Juhani Pietarinen, "Semantic Information and Inductive Logic," in J. Hintikka and P. Suppes eds., Aspects of Inductive Logic (Amsterdam: North Holland, 1966), Pp. 96-112, and Risto Hilpinen, Rules of Acceptance and Inductive Logic (Amsterdam: North Holland, 1968), pp. 103-22. Carl G. Hempel, Aspects of Scientific Explanation (New York: Free Press, 1965), pp. 73-78. 
However, the importance of arriving at true conclusions, if not the only goal, is in a way a supervening one. For, conclusions that are false do not add to our information nor do they facilitate explanation. No matter how informative a statement would be if it were true, we shall be misled rather than enlightened if we accept it when it is false. Similarly, what explains must be true or the explanation fails, and what is explained must be a fact, something described by a true statement not a false one, or else there is nothing to explain.

A proper concern for truth may be guaranteed by requiring that the conclusions of inductive reasoning should be more probable than the premises of any argument sufficient to demonstrate the falsity of the hypothesis. Thus we shall restrict inductive inference so that there is no acceptable argument demonstrating the falsity of an inferred hypothesis. Grice, Chisholm, and Thomas Reid have suggested that some hypotheses may be regarded as innocent until proven guilty. ${ }^{5}$ Others, notably Popper and his followers, have suggested that the strongest hypothesis consistent with the evidence is the one to accept. ${ }^{6}$ Both of these theses incorporate the idea that there must not be any acceptable argument demonstrating the falsity of a hypothesis inferred from background knowledge.

A hypothesis may be inductively inferred from background knowledge only if there is no acceptable argument demonstrating the falsity of the hypothesis. Demonstrative arguments must be deductive. Moreover, among deductive arguments we may restrict our consideration to relevant ones for reasons cited above. Thus, an argument is an acceptable demonstrative argument against a hypothesis only if it is a relevant deductive argument whose conclusion contradicts the hypothesis under consideration. But this is not the only condition that an argument must satisfy in order to be an acceptable demonstrative argument against a hypothesis. For, there remains the question of the viability of the premises of such an argument. The premises of the argument must each be at least as probable on the basis of the background knowledge as the hypothesis under consideration or else the argument is unacceptable as a demon-

\footnotetext{
${ }^{5}$ H. P. Grice, "The Causal Theory of Perception," in Robert J. Swartz ed., Perceiving, Sensing and Knowing (Garden City, N. Y.: Doubleday, 1965), pp. 470-71. R. M. Chisholm, Perceiving: A Philosophical Study (Ithaca, N. Y.: Comell University Press, 1957), p. 9. Thomas Reid, The Works of Thomas Reid, D.D. (Edinburgh: Maclaugh and Steward, 1863), p. 234.

${ }^{\circ}$ Popper, The Logic of Scientific Discovery, 57-90.
} 


\section{Keith Lehrer}

stration of the falsity of the hypothesis. If the hypothesis in question is more probable on the background knowledge than one of the premises of the argument, then the argument is not an acceptable argument against the hypothesis. The reason is that a relevant argument is one in which all the premises are essential for the deduction of the conclusion, and consequently such an argument is only as acceptable as its weakest premise.

5. An Inductive Rule. On the basis of these considerations, it is contended that a hypothesis may be inductively inferred from background knowledge if and only if the hypothesis is such that any relevant deductive argument whose conclusion is the contradictory of the hypothesis is such that at least one premise of the argument is less probable on the background knowledge than the hypothesis inferred. The rule may be expressed in terms of the notion of a minimally inconsistent set as follows: a hypothesis may be inductively inferred from background knowledge if and only if every minimally inconsistent set to which it belongs has at least one other member which is less probable on the background knowledge than the hypothesis.

This very abstract characterization of an inductive inference has features that make it suitable as a principle of rationality. For example, the set of hypotheses that may be inductively inferred from background knowledge by such a rule must be logically consistent as a set and the set must be consistent with the background knowledge. The proof is that if we assume that the set $S$ of hypotheses inferred from the background knowledge is logically inconsistent, then, since each of the hypotheses belonging to the set is consistent, it follows that some subset of $S$ having at least two members must be a minimally inconsistent set. Since each of the two members was inferred by the rule, each member of the minimally inconsistent set must be more probable than at least one other member. But this is impossible. Hence the original set of inductively inferred hypotheses must be logically consistent.

This consistency feature is lacking in some plausible alternatives. The reason is that a set of hypotheses having any probability less than one on background knowledge can always be so selected that the set is inconsistent with the background knowledge. If someone adopts a rule to the effect that any hypothesis having a probability greater than $\mathrm{m} / \mathrm{n}$ ( $\mathrm{m}$ being a smaller integer than $n$ ) on background knowledge may be inductively inferred from that knowledge, then, as Kyburg has shown, he 
will be selecting a rule that will permit him to inductively infer a set of hypotheses from background knowledge that is inconsistent with that knowledge. ${ }^{7}$ For example, suppose he has a fair lottery and background knowledge saying that of $\mathrm{n}$ consecutively numbered tickets, exactly one is the winner. In this case, the probability that it is not the number one ticket is at least $m / n$, the probability that it is not the number two ticket that wins is at least $m / n$, and so forth. Thus, the set of inferred hypotheses would be such that one could deduce from the set that no ticket is the winner. This contradicts the background knowledge that one ticket is the winner. Thus the consistency of the inferred hypotheses with the background knowledge is a significant feature of the rule. If one regards it as rational to infer a consistent rather than an inconsistent set of conclusions from what one knows, then the rule fulfills a requirement of rationality.

To formulate other results of applying the rule, it will be useful to have a more formal statement of it. Letting " $I(h, b)$ " mean " $h$ may be inductively inferred from $b$," the rule is as follows:

I. $I(h, b)$ if and only if for any set $S$ of statements of $L$, if $h$ is a member of $S$ and $S$ is minimally inconsistent, then there is a $k$, $k \neq h$, such that $k$ is a member of $S$ and $P(h, b)$ exceeds $P(k, b)$.

The probability functor is a relation between the statements " $h$ " and " $b$ " which satisfies the calculus of probability. We may think of the concept of probability as being interpreted in the manner explicated by Carnap or Hintikka, but other analyses would be appropriate as well. ${ }^{8}$ Moreover, we shall limit our discussion to finite languages which may be defined in terms of what Levi calls an ultimate partition, a partition being a set of statements that are exclusive in pairs and exhaustive as a set. ${ }^{\text {T }}$ The ultimate partition may also be thought of as defining the language in the sense that any statement of the language is logically equivalent to a disjunction of members of the partition or to a single member of a partition or to conjunction of a member and its denial. The state-descriptions are, of course, members of a partition in Carnap's

${ }^{7}$ H. E. Kyburg, Jr., Probability and the Logic of Rational Belief (Middletown, Conn.: Wesleyan University, 1957), p. 197.

${ }^{8}$ Rudolph Camap, The Foundations of Probability (Chicago: University of Chicago Press, 1950), and Jaako Hintikka, "A Two-Dimensional Continuum of Inductive Methods," in Hintikka and Suppes, eds., Aspects, pp. 113-32.

${ }^{\circ}$ Levi, Gambling with Truth, pp. 32-36. 


\section{Keith Lehrer}

system. Languages without individual constants may be thought of as being defined by a partition, only in this case the partition will not be a set of state-descriptions. Finally, let it be noticed that a partition can be generated from any set of statements. If one has a set of statements, $h l, h 2$, and so forth, then one can construct a partition by forming maximal conjunctions such that each $h_{i}$ or its negation (but not both) is contained in order in each maximal conjunction. When one thus obtains a set of such conjunctions containing all possible permutations and then eliminates those maximal conjunctions that are inconsistent, the remaining set of maximal conjunctions will be a partition.

6. Results of the Inductive Rule. With the foregoing technical remarks on the board, we may then ask what sort of results (I) yields. The results may be succinctly characterized. A disjunction of members of the ultimate partition may be inductively inferred from background knowledge if and only if the probability of the hypothesis on background knowledge exceeds the probability of the denial of at least one disjunct on the same knowledge. ${ }^{10} \mathrm{~A}$ single member of the ultimate partition may be inductively inferred from background knowledge if and only if it is more probable than its denial on background knowledge. ${ }^{11}$ Only (but not all) those hypotheses having a probability greater than $1 / 2$ on background knowledge will be ones that can be inductively inferred from background knowledge. ${ }^{12}$

We obtain a more concrete idea of the results by again considering lotteries. In the fair lottery, we may not inductively infer that the number one ticket will not win, because this hypothesis may be considered as a disjunction of the hypotheses saying that either the number two ticket or the number three ticket and so forth will win. The denial of each of these disjuncts is equal in probability to that of the hypothesis that the number one ticket will not win. Hence the probability of the hypothesis does not exceed the probability of the denial of any disjunct contained in it.

Now let us consider some skewed lotteries where the tickets have differing probabilities of being the winner. In the event that it is more probable that a ticket will win than lose, we may inductively infer the

${ }^{10}$ See T6 in the appendix for a proof of this result.

${ }^{11}$ See T5 in the appendix for a proof of this result.

12 The proof of this hypothesis follows from the theorem that a hypothesis having a probability no greater than $1 / 2$ has no greater probability than its denial. 
hypothesis that the ticket will win from the background knowledge. If there is no ticket such that the probability of its winning exceeds the probability of its losing, then we cannot inductively infer that any specific ticket will win. However, we may inductively infer from the background knowledge that some ticket will not win or that one or the other of some group of tickets will win. We may infer that a ticket will not win if there is any other ticket that has a higher probability of winning on the background knowledge. We may infer a disjunction asserting that one of a specified group of tickets will win if it is more probable that one of the group will win than it is that at least one specific one of the tickets of the group will lose. Thus, for example, one may inductively infer that the winning ticket will be one of the first ten if that hypothesis is more probable than the hypothesis that the number one ticket will lose, or more probable than that the number two ticket will lose, or ... more probable than that the number ten ticket will lose.

The foregoing results have been expressed in terms of lotteries for the sake of clarity. However, it should not be concluded that the inductive method in (I) is therefore limited to such contexts. On the contrary, whenever hypotheses are under scientific consideration, we may construct a partition from those hypotheses in the manner indicated above, the hypotheses then being equivalent to a single member of the partition or to some disjunction of such members, and then apply the inductive rule. The set of hypotheses must be finite, but this is not as restrictive as it might appear. As Hempel has argued, even such a simple problem as determining the length of some object by measurement allows for an infinite number of different results, and such considerations might appear to indicate that (I) will have very limited applicability. ${ }^{13}$ But this conclusion would be precipitous. Though it is mathematically true that an object may have any of an infinity of different lengths, in fact any procedure of measurement will have some least measure, and, thus once we set some limits to the length the object might have, even very rough limits, the measurement problem becomes amenable to the application of (I). If we suppose that $m$ is the least measure of our measurement procedure and find a number $m n$ ( $n$ being an integer) such that the probability that the length is at least $m n$ is equal to one, then we may

${ }^{13}$ Carl G. Hempel, "Fundamentals of Concept Formation in Empirical Science," in Otto Neurath ed., International Encyclopedia of Unified Science, vol. 2, no. 7 (Chicago: University of Chicago Press, 1952), pp. 29-35. 


\section{Keith Lehrer}

restrict our consideration to a set of hypotheses affirming that the length of the object is $m n+m, m n+2 m, m n+3 m$, and so forth until we reach a hypothesis such that the probability of the length exceeding that number is zero. We will then have a partition to which IR may be applied.

Another apparent problem dealing with the application of $I R$ in a scientific context concerns the inference of general hypotheses and theories. General hypotheses, though often inconsistent with each other, do not seem to form a partition because one rarely has an exhaustive set to consider. However, this difficulty can be easily overcome by adding what Shimony has called a remainder hypothesis, namely, a hypothesis affirming that none of the other hypotheses are correct. ${ }^{\mathbf{1 4}}$ If the other hypotheses are incompatible in pairs, then those hypotheses together with the remainder hypothesis will constitute a partition. If the others are not inconsistent in pairs, we may generate a partition by employing the procedure of forming maximal conjunctions of the hypotheses, including the remainder hypothesis, and rejecting the inconsistent conjunctions. The remaining set will be a partition. We can then apply IR to decide which hypotheses should be inferred.

It is clear from these remarks that the selection of the partition is of fundamental importance. It is tempting simply to cast this problem into the pit of pragmatics, and say, with Levi, that the selection of an ultimate partition is a pragmatic choice. ${ }^{15}$ However, this approach obscures important issues. Certain selection procedures would be arbitrary and unreasonable. Moreover, the selection of two different ultimate partitions, two different languages, both of which are used at the same time, could lead to the inference of inconsistent results by (I) even though the application (I) to a single partition is consistent. Thus, to retain the consistency feature of (I) in application, one must restrict the choice of ultimate partitions. Some philosophers, who stress the relation of scientific advance to conceptual innovation, will regard the selection of ultimate partitions as constituting a basic feature, if not the basic feature, of scientific inquiry. At any rate, the problem of selecting a language, an ultimate partition, if pragmatic, must be so restricted as to conform to reasonable standards of scientific procedure. The selection must not be arbitrary, it must not lead to the simultaneous selection of a multi-

${ }^{14}$ Abner Shimony, essay in this volume.

${ }^{18}$ Levi, Gambling with Truth, pp. 67-68, 91-95. 


\section{INDUCTION, RATIONAL ACCEPTANCE, AND SETS}

plicity of different partitions that yield conflicting results, and it must not fail to meet other standards of cogency, completeness, and simplicity. Thus, the choice of a language or ultimate partition which is required for the application of (I) raises some of the most important issues in the philosophy of science. However, even once a choice has been made concerning the language or ultimate partition and the evidence or background knowledge, there remains the problem of deciding what hypotheses in that language or partition may be inferred from that evidence or knowledge. We may attempt to solve that problem without pretending to resolve those issues concerning what languages to select or what statements to consider as background knowledge.

One final problem of application concerns the assignment of probabilities. It might be thought that it is not feasible to suppose that one can meaningfully assign degrees of probability to all hypotheses in all scientific contexts. The reply to this line of objection is twofold. First, developments in subjective theories of probabilities owing to Savage and Jeffrey suggest that probabilities may be assigned on the basis of empirically determined preferences where initially assignment of probabilities might appear impossible or arbitrary. ${ }^{16}$ So, even if the logical theories of Carnap and Hintikka prove inadequate in some cases, there remains the possibility of appealing to subjective theories or appropriate modifications thereof. Second, the rule (I) only requires that we be able to make comparative judgments of probabilities, that we be able to determine whether two hypotheses are such that one is more probable, less probable, or equal in probability to another. Thus, although I have employed a quantitative concept of probability in the formulation of the rule, a comparative concept of probability could replace the quantitative one for the application of IR in contexts in which only comparative judgments of probability are feasible. As so modified, the rule would have broad application, because such comparative judgments would be feasible in a wide variety of scientific contexts.

7. The Rationality of the Inductive Rule. The next task is to show that these results are rational, that is, that it would be reasonable to infer inductively such hypotheses from background information according to

${ }^{10}$ Leonard J. Savage, "Implications of Personal Probability for Induction," Journal of Philosophy, 64 (1967), 593-607, and The Foundations of Statistics (New York: John Wiley, 1954). Richard Jeffrey, The Logic of Decision (New York: McGrawHill, 1965). 


\section{Keith Lehrer}

(I). We have already noted that the set of statements inductively inferred from background knowledge by (I) will be logically consistent with the background knowledge. Secondly, the rule has the feature of restricted deductive closure. This principle may be formulated as follows:

PRDC. If $I(h, b)$ and $k$ is a deductive consequence of $h$, then $I(k, b) \cdot .^{17}$

This principle of restricted deductive closure tells us that for any hypothesis that may be inductively inferred from background knowledge, a deductive consequence of the hypotheses may likewise be inferred. And again this is a desideratum of rationality in that it would be irrational to refuse to infer inductively a deductive consequence of a hypothesis one had inductively inferred. ${ }^{18}$

Thirdly, (I) has a claim to being a principle of rationality because anything that one infers will be such that one can always offer a probabilistic reason for the inference, and for any inference one cannot make one also has a probabilistic reason for not making it. Consider, for example, members of the ultimate partition. If one infers that such a member is false, it will always be the case that some other member was more probable. If one infers that some member is true, then it will always be the case that it was more probable than its denial and also more probable than any other member. And if one cannot infer that a member is true, then it will be at least as probable that some other member is true instead and that the member is false rather than true. With respect to disjunctions, if one infers that a disjunction is true, then it will be more probable that the disjunction is true than it is that at least one of its disjuncts is false and also more probable that it is true than false. If one cannot infer that a disjunction is true, then it will be at least as probable that each of its disjuncts is false as it is that the disjunction is true.

8. Induction and Explanation. Moreover, when the rule is applied to the appropriate ultimate partitions, the inferred hypotheses will, if true, explain certain facts contained in the background knowledge and others as well. Hintikka and Hilpinen have shown that partitions of general hypotheses describing the kinds of individuals there are in the universe

\footnotetext{
${ }^{17}$ See $\mathrm{T} 7$ in the appendix for proof of this result.

${ }^{18}$ H. E. Kyburg, Jr., "Probability, Rationality, and a Rule of Detachment," in Y. Bar-Hillel ed., Proceedings of the 1964 Congress for Logic, Methodology and Philosophy of Science (Amsterdam: North Holland, 1965), pp. 303-10.
} 
are such that, when probabilities are assigned by Hintikka's methods, the probability that all unobserved individuals are of the same kind as those observed goes up sharply as the number of observed individuals increases..$^{19}$ When the probability of one such hypothesis exceeds $1 / 2$ and thus exceeds the probability of any other hypothesis belonging to the ultimate partition, then by (I) the hypothesis may be inferred. Such a hypothesis could be employed to explain why we have observed the kinds of individuals we have and not other kinds, to wit, because all individuals are of the kinds we have observed and there are no other kinds. When we seek to explain why all individuals are of the kinds they are by appealing to higher level theories, we may again apply (I) to enable us to decide which theory we may infer, provided we can make comparative judgments concerning the probability of such theories. At both levels, the application of (I) will facilitate explanation.

We may also consider whether (I) is itself a principle of inductive explanation, that is, whether we may conclude that a hypothesis inductively inferred from background knowledge by (I) is also inductively explained by that knowledge. I have elsewhere argued that a more restrictive inductive rule is required to ensure that the hypothesis is inductively explained by the probabilities. ${ }^{20}$ The reason is that by (I) it may turn out to be the case that two hypotheses are inferred, the first more probable than the second, when the first turns out to be false and the second true. For example, suppose that we have a three-membered ultimate partition whose members $h l, h 2$, and $h 3$ have probabilities of $1 / 6$, $2 / 6$, and $3 / 6$ respectively. By (I) we may infer $\sim h l$ and $\sim h 2$. However, if $h 1$ turns out to be true, it would be peculiar to say that the truth of $\sim \mathrm{h} 2$ is explained by the probabilities, because $\sim \mathrm{hl}$ was even more probable and it turned out to be false. It is possible to formulate a more restrictive inductive rule than (I) having the consequence that an inferred hypothesis will, if it is true, be more probable than any hypothesis that turns out to be false. But (I) lacks this feature. Nevertheless, we may say of hypotheses inductively inferred from background knowledge (and not deducible from such knowledge) by (I) that they are potentially explained by the background knowledge, because if all equally

${ }^{19}$ Jaako Hintikka and Risto Hilpinen, "Knowledge, Acceptance, and Inductive Logic," in Hintikka and Suppes, eds., Aspects, Pp. 1-20; and see Hilpinen, Rules, PP. $50-67$.

${ }^{20}$ Lehrer, "Justification," pp. 110-27. 


\section{Keith Lehrer}

probable or more probable hypotheses inferred from such knowledge by (I) turn out to be true, then the truth of such hypotheses together with the background knowledge does inductively explain the truth of the hypotheses inferred. It is explained by the probabilities.

The foregoing considerations imply that if one seeks to reach conclusions in scientific inquiry which explain what needs to be explained and are themselves explained, it is reasonable to apply (I) to obtain these objectives. For, given certain ultimate partitions, the hypotheses inductively inferred from background knowledge by (I) will explain facts contained within the background knowledge as well as other facts, and hypotheses inductively (but not deductively) inferred from background knowledge by (I) are potentially explained by the probabilities. 9. A Rule of Acceptance. However, a more direct proof of the rationality of the rule is possible. Some philosophers, Levi and Kyburg for example, have argued that rules of inductive logic may be considered as directives to accept hypotheses on the basis of background knowledge. According to this account, inference aims at acceptance. We may regard anything inductively inferred from background knowledge as suitable to be accepted on the basis of that knowledge, but there are other requirements that must be met by a theory of acceptance. One of these, Levi has argued, is a principle of deductive cogency according to which the set of accepted statements includes the deductive consequences of the background knowledge and accepted hypotheses. ${ }^{21}$ In short, the set of accepted statements must be deductively closed. The reason for this is that it would be irrational for a man to refuse to accept the deductive consequences of the statements he accepts.

In order to provide a theory of acceptance built upon our inductive rule we shall say that a hypothesis is to be accepted on the background knowledge if it may be inductively inferred from background knowledge or may be deduced from some set of hypotheses that may be inductively inferred from that knowledge. Formally stated, we obtain the following rule where we let " $A(h, b)$ " mean " $h$ may be accepted on the basis of $b . "$

AR. $A(h, b)$ if and only if either (i) $I(h, b)$ or (ii) there is some

${ }^{2}$ Levi, Gambling with Truth, pp. 26-28. H. E. Kyburg, Jr., has argued against this principle in "Conjunctivitis," in Swain, ed., Induction, Acceptance, and Rational Belief, pp. 55-82. 
set $S$ of which $h$ is a deductive consequence and such that $k$ is a member of $S$ if and only if $I(k, b)$.

This rule is deductively closed; that is, the set of statements that may be accepted relative to background knowledge includes its own deductive consequences.

The results that we obtain by employing (AR) are very easy to characterize. If there is some member of the ultimate participation that is more probable on background knowledge than any other member of the ultimate partition, then the set of hypotheses we may accept consists of the logical consequences of that hypothesis and the background knowledge. This follows from the fact that we may inductively infer that each of the other members is not true by (I), and the conjunction of those logically implies that the remaining member of the partition is true. If there is some set of members of the ultimate partition that are equally probable and more probable than any member not belonging to the set, then the set of statements we may accept will consist of the logical consequences of the disjunction of those equally probable members and the background knowledge. This follows from similar considerations, namely, that we may inductively infer the falsity of each of the members of the ultimate partition not belonging to the set of members having the highest probability. ${ }^{22}$

10. The Objectives of Acceptance. The next question to confront is, How we are to prove that such an acceptance rule as (AR) is a principle of rationality? Levi has argued that a policy of accepting a statement is rational if and only if it can be shown to satisfy the objectives of acceptance. ${ }^{23}$ To decide whether it is rational to accept a hypothesis we must first determine what aims or goals we hope to achieve by accepting hypotheses. One set of goals is that of accepting hypotheses that are true and informative. If we restrict ourselves to aiming at accepting true hypotheses, it would never be rational to venture beyond accepting the deductive consequences of our background knowledge. For, as we said earlier, there is always some risk of accepting a false hypothesis whenever one accepts any hypothesis that is inductively inferred from such knowledge, and, such a risk would not be rational if our only interest were to accept hypotheses that are true. Crudely put,

${ }^{20}$ See T8 in the appendix for a proof of this result.

${ }^{23}$ Levi, Gambling with Truth, pp. 7-19. 


\section{Keith Lehrer}

it is not just truth that we desire but also the whole truth or as much of the whole truth as we can get. The more content our accepted statements have the more of the truth we get if such statements are true. The more content a statement has the more informative it is. Thus, we may, with Levi, regard truth and content as objectives of acceptance. 11. Maximizing Expected Utility. We may now show that AR aims at the satisfaction of these objectives. Following Hempel, Hintikka, and Levi, we formulate some utility function and then show that, where we have the twin objectives of truth and content, a policy of maximizing expected utility yields the same results as (AR). The notion of maximizing expected utility is, informally, the following. First one considers the alternatives before one and the value or utility one attaches to the possible outcomes of adopting the various alternatives. One also has to consider how probable it is that the selection of a given alternative will produce each outcome. The expected utility of selecting a given alternative is the sum that one gets from adding the products of the probability of each outcome on that alternative times the utility of that outcome.

When applying this technique to matters of acceptance, we may restrict consideration to a pair of possible outcomes. When we accept $h$ either we accept $h$ and $h$ is true, that is, we correctly accept $h$, or we accept $h$ and $h$ is false, that is, we incorrectly accept $h$. To put the matter more formally, we have only to define two utilities, the utility of accepting $h$ when $h$ is true and the utility of accepting $h$ when $h$ is false.

To render the preceding considerations more precise, it will be useful to introduce some formal notation derived from Levi. First, letting " $E(h, b)$ " mean "the expected utility of accepting $h$ on $b$," "U(h,b)" mean "the utility of correctly accepting $h$ on $b$," and " $u(h, b)$ " mean "the utility of incorrectly accepting $h$ on $b$," we obtain the following equation:

$$
E(h, b)=P(h, b) U(h, b)+P(\sim h, b) u(h, b) .{ }^{24}
$$

Given this formulation of expected utility, and given that we agree that the rational course of acceptance is to maximize expected utilities, all we need to do in order to determine whether our acceptance is rational is to define the utilities and a notion of maximality. Let us first ${ }^{2}$ Cf. ibid., p. 83, and Hempel, Aspects, pp. 73-78. 
consider the matter of defining the utilities in question.

12. Maximizing Truth and Content. Suppose that we are seeking to maximize the utilities of truth and content. In the equation, the probability of $h$ on $b$ and the probability of $\sim h$ on $b$ are given equal weight with the utility. Probability is the probability of truth. Thus the objective of truth is given equal weight to that of the utility. Consequent$\mathrm{ly}$, in defining the utilities in question, we may be satisfied if they represent the objective of content. It might in fact seem that we could simply identify the utility of correctly accepting $h$ on $b$ when $h$ is true with the content of $h$. Letting "cont $(h, b)$ " mean "the content of $h$ on $b$," we would then obtain the equation

$$
U(h, b)=\operatorname{cont}(h, b)
$$

and similarly

$$
u(h, b)=-\operatorname{cont}(-h, b) .^{25}
$$

These equations, a simplification of ones proposed by Levi, embody the idea that if we accept $h$ when $h$ is true, then we obtain the full benefit of the information contained in $h$, and if we accept $h$ when $h$ is false, then we suffer the full loss of not having the information contained in $\sim$ h.

The foregoing is very plausible, but the question of whether it is adequate can only be resolved once we have produced some method for assigning content value. One way of doing this, again suggested by Levi, is to assign equal content values to each of the members of the ultimate partition. Where there are $n$ members in the partition we might accomplish this by defining a regular measure function $m$ on the members $\mathrm{kl}, \mathrm{k} 2, \ldots, \mathrm{kn}$, as follows:

$$
\mathrm{m}\left(\mathrm{k}_{\boldsymbol{i}} b\right)=1 / \boldsymbol{n}
$$

and where $d$ is a disjunction of $k_{i} s$ each appearing but once and having $r$ disjuncts

$$
m(d, b)=r / n .
$$

On the basis of this measure function we define content as follows:

$$
\operatorname{cont}(h, b)=1-m(h, b) .^{26}
$$

${ }^{25}$ Cf. Levi, Gambling with Truth, p. 80.

${ }^{28}$ Cf. ibid., pp. 69-70. 


\section{Keith Lehrer}

The rationale behind this assignment is a pragmatic one, to wit, that each of the members of the ultimate partition is equally a good answer to a problem that leads to the choice of the ultimate partition and hence each member of the ultimate partition is equally informative.

There are other ways of assigning content, but on the basis of this assignment we find some reason for rejecting the equation of utilities with content in the manner outlined. I have argued elsewhere that doing so leads to an insensitivity to the differences of probability between members of the ultimate partition and to a certain indifference to accepting hypotheses of high content. The latter can be simply illustrated. If one values high content over low, then the utility of accepting $h$ when $h$ is true should not be equated with the content of $h$, because that would not show a strong preference for high content over low content. For example, suppose that one has a partition of ten members. In that case, the content of a member of the partition is $9 / 10$ while a disjunction of two different members has a content of $8 / 10$, only slightly less. However, if one is aiming at accepting highly informative conclusions, then one should value accepting a single member twice as much as accepting such a two-membered disjunction, because the latter is only half as informative as the former. Similarly, one should value accepting a member three times as much as a three-membered disjunction of different members, four times as much as a four-membered disjunction, and so forth. In short, one should value the content of a member $\boldsymbol{n}$ times as much as a disjunction of $\boldsymbol{n}$ different members.

We may obtain such results by defining the utilities not only in terms of content, but also in terms of a number $n_{h}$ equal to the number of different members of the ultimate partition occurring in a disjunction of members which is equivalent to $h$. If $h$ is a member of the ultimate partition, then $n_{h}$ equals 1 . This proposal is expressed in the following equations:

$$
\begin{aligned}
& U^{*}(h, b)=\operatorname{cont}(h, b) / n_{h} . \\
& u^{*}(h, b)=-\operatorname{cont}(\sim h, b) / n_{h} .
\end{aligned}
$$

From these equations we obtain the following equalities:

$$
\begin{aligned}
& E(h, b)=P(h, b)-\operatorname{cont}(\sim h, b)) / n_{h} . \\
& E(h, b)=P(h, b)-\operatorname{cont}(\sim h, b)
\end{aligned}
$$

if $h$ is a member of the ultimate partition. 


$$
\left.E(h, b)=\underset{i=1}{j} \sum_{i=1}^{j} E\left(k_{i}, b\right)\right) / n_{h}
$$

if $h$ is equivalent to a disjunction of the set of members $k 1, k 2, \ldots$, kj of the ultimate partition. ${ }^{27}$

Thus, we may now determine which hypotheses it is rational to accept by defining maximality. With respect to language $L$, we may, again following Levi, define two notions of maximality:

$E(h, b)$ is maximal in $L$ if and only if, for every $s$ in $L, E(s, b)$ is no greater than $E(h, b)$.

$E(h, b)$ is strongly maximal in $L$ if and only if $E(h, b)$ is maximal, and for every $s, s \neq h$, such that $E(s, b)$ is maximal in $L$, cont$(h, b)$ is less than cont $(s, b) .{ }^{28}$

The notion of strong maximality is introduced to tell us what to accept in cases of ties. It tells us, among other things, that if the expected utility of each of a set of members of the ultimate partition is maximal, then the disjunction of those members is strongly maximal. We can now formulate a rule for maximizing expected utility having deductive closure as follows:

(R) $A(h, b)$ if and only if either (i) $E(h, b)$ is strongly maximal or (ii) $h$ is a deductive consequence of the set $S$ which has as its only members $b$ and $k$ where $E(k, b)$ is strongly maximal.

Rule (R) formulated as a principle for maximizing utilities that aim at accepting true and informative statements is equivalent in the results it yields to the earlier principle (AR). ${ }^{29}$ Thus (AR) is a principle of rationality for the purpose of obtaining the objectives of truth and content.

13. Summary. We began with the concept of a relevant deductive argument analyzed as an argument in which the denial of the conclusion together with the premises constitutes a minimally inconsistent set of statements. In terms of this concept we defined an acceptable argu-

${ }^{27}$ Keith Lehrer, "Induction: A Consistent Gamble," Nous, 3 (1969), 290-94.

${ }^{23}$ Levi, Gambling with Truth, p. 83.

${ }^{2}$ See T8, T9, and T10 in the appendix for proof of this result. 


\section{Keith Lehrer}

ment demonstrating the falsity of a hypothesis as a relevant deductive argument in which the conclusion is the contradictory of the hypothesis in question and the premises are each at least as probable as the hypothesis on background knowledge. An inductive rule was then proposed: a hypothesis may be inductively inferred from background knowledge if and only if there is no acceptable argument demonstrating the falsity of the hypothesis. It was shown that this inductive rule meets various conditions of rationality. First, the set of conclusions inductively inferred from background knowledge by the rule must be logically consistent with that knowledge. Second, any deductive consequence of a hypothesis inductively inferred by the rule could itself be inductively inferred by the rule from the same knowledge. Third, any application of the rule is such that there is a probabilistic reason for inferring what is inferred by the rule. Fourth, partitions may be so chosen that hypotheses inductively inferred by the rule will, if true, explain facts contained within background knowledge and others as well. Moreover, a hypothesis that is inductively (and not deductively) inferred from background knowledge is potentially explained by the probabilities. Finally, an acceptance rule based on the inductive rule yields the acceptance of hypotheses equivalent to adopting the policy of maximizing expected utility in the acceptance of hypotheses when what one values is truth and informational content.

The accceptance rule articulated and defended recommends a very adventurous policy of inductive inference. In effect, it tells us that whenever our background knowledge favors a strong hypothesis over others of equal strength, where strength is a measure of informational content, then we should inductively infer that hypothesis. It gives us this direction no matter how slightly the background knowledge favors the hypothesis in question. Some might consider such a policy too risky and advocate some more cautious policy instead. However, in my opinion, the history of science reveals that scientific inquiry has advanced more significantly through rational but adventurous attempts to obtain new information than through safe and cautious attempts to avoid error. It is for that reason that the acceptance rule is proposed as a principle of rational acceptance for the explication of scientific reasoning. ${ }^{30}$

so Work on the present article was supported by a National Science Foundation Grant. Other articles written under this grant on the subject of induction by Keith Lehrer include "Induction and Conceptual Change," Synthese, 23 (1971), 206-25, 


\section{Appendix}

The purpose of this appendix is to prove various theorems assumed in the main text. The proofs offered are only intended to constitute a sketch of a completely formalized proof, but one from which the latter could be constructed quite mechanically. All theorems are proven for a finite language $L$ which consists of an ultimate partition, that is, a set of statements $k l, k 2, \ldots, k n$ that are disjoint in pairs and exhaustive as a set, and truth functional combinations of such statements. I assume the following calculus of probability for such languages, letting " $p(h, s)$ " mean "the probability of $h$ on $s$," on the condition that the statement to the right of the comma in the probability expression is logically consistent:

P1. $0 \leq \mathrm{p}(h, b) \leq 1$.

$P 2$. If $b$ is logically equivalent to $b^{\prime}$ and $h$ is logically equivalent to $h^{\prime}$, then $p(h, b)=p\left(h^{\prime}, b^{\prime}\right)$.

P3. $p(h, b)=1$ if and only if $h$ is a deductive consequence of $b$.

P4. If $\sim(h \& k)$ is a deductive consequence of $b$, then $p(h \vee k, b)$ $=p(h, b)+p(k, b)$.

P5. $p(h \& k, b)=p(h, b) \times p(k, h \& b)$.

The following theorems are required to establish various results concerning the rules of induction and acceptance. Let $k 1, k 2, \ldots, k n$ be the $n$ members of the ultimate partition $P$ and let $G$ be a set consisting of the members of $P$ and disjunctions of such members.

T1. If $k$ is a disjunction of $n-1$ different members of $P$, then $k$ is a consequence of a set $S$ of members of $G$ only if $k$ is a deductive consequence of some member of $S$. Proof: If $k$ is a disjunction of $n-1$ different members of $P$, then there is only one member of $P$ that is not a disjunct of $k$. Let $h$ be that one member. Any disjunction of members of $P$ not containing $h$ as a disjunct will have $k$ as a deductive consequence. Hence if a set of members of $G$ has $k$ as a deductive consequence but no single member of the set has $k$ as a deductive consequence, then all members of the set must either contain $h$ as a disjunct or else be $h$ itself. But such a set of statements would all be true if $h$ were true even though $k$ would then be false. Therefore, $h$ is not a deductive consequence of such a set.

and "Reasonable Acceptance and Explanatory Coherence: Wilfrid Sellars on Induction," Nous, 7 (1973), 81-103. 


\section{Keith Lehrer}

T2. If $d$ is a disjunction of members of $P$, then any minimally inconsistent set $M$ of which $d$ is a member will be such that once $d$ is deleted from $M$ the remaining set $R$ of statements will have the negations of each disjunct of $d$ as deductive consequences. Proof: Suppose there was some denial of a disjunct of $d$ that was not a deductive consequence of $R$. In that case, the members of $R$ could all be true (all proper subsets of $M$ are consistent) when $d$ was also true because that disjunct is true whose negation is not a consequence of $R$. Hence the set $M$ would not be inconsistent.

T3. For any $k$ and $b$, if $k$ is a member of $P$, and $d$ is a disjunction of all the remaining members of $P$, then there is no member $m$ of $G$ of which $\sim k$ is a deductive consequence such that $p(m, b)$ is greater than $p(d, b)$. Proof: The disjunction $d$ is logically equivalent to $\sim k$. Moreover, from $\mathrm{P} 1, \mathrm{P} 3$, and $\mathrm{P} 4$ of the calculus of probability it follows that for any statement $s$ that is a deductive consequence of $h, p(s, b)$ is at least as great as $p(h, b)$. Therefore, if $d$ is a deductive consequence of any member $m$ of $G$, it follows from the preceding result and $P 2$ in calculus of probability that $p(\mathrm{~m}, b)$ is not greater than $p(d, b)$.

T4. For any disjunction $d$ of two or more members of $P$, and for any minimally inconsistent set $S$ of statements whose members are also members of $G$ and include $d$, and for any negation $n$ of a disjunct of $d$, there is some member $m$ of $S$ such that $p(m, b)$ is no greater than $p(n, b)$. Proof: If $d$ is a disjunction of two or more members of $P$ and $k$ is a disjunct of $d$, then $\sim k$ must be a deductive consequence of some member $m$ of any minimally inconsistent set $S$ of members of $G$ that includes $d$ by $\mathrm{T} 1$ and $\mathrm{T} 2$. From $\mathrm{T} 3$ it follows that for any such disjunct $k$ and member $m$ of such an $S, p(m, b)$ is not greater than $p(\sim k, b)$.

The next theorems concern the inductive rule (I) which is as follows:

I. $I(h, b)$ if and only if for any set $S$ of statements of $L$, if $h$ is a member of $S$ and $S$ is minimally inconsistent, there is a $k, k \neq h$, such that $k$ is a member of $S$ and $p(h, b)$ exceeds $p(k, b)$.

It is a theorem of combinatorial logic that every noncontradictory member of $L$ is logically equivalent to some member of $G$. Since no contradictory statement of $L$ belongs to any minimally inconsistent set of statements having more than one member; since logically equivalent statements have the same probability on the same statement by P2; and since any minimally inconsistent set from which one statement is de- 
leted and a logically equivalent statement added will remain a minimally inconsistent set, the set of statements $C$ such that $h$ is a member of $C$ if and only if $I(h, b)$ by $(I)$ is logically equivalent to the set of statements $C^{\prime}$ such that $h$ is a member of $C^{\prime}$ if and only if $I^{\prime}(h, b)$ by $\left(I^{\prime}\right)$ as follows:

$\left(I^{\prime}\right) . I^{\prime}(h, b)$ if and only if for any set $S$ of statements of $G$ of $L$, if $h$ is a member of $S$ and $S$ is minimally inconsistent, then there is a $k, k \neq h$, such that $k$ is a member of $S$ and $p(h, b)$ exceeds $p(k, b)$.

Hence, in the subsequent proofs concerning (I) we may in some proofs restrict our consideration to statements of $G$ for the sake of simplicity without any significant lack of generality.

T5. If $h$ is a member of $P$, then $I(h, b)$ if and only if $p(h, b)$ exceeds $p(\sim h, b)$. Consider a set $S$ of members of $G$ that includes $h$ which is a member of $P$ and is minimally inconsistent. $S$ must have some member $m$ of which $\sim h$ is a deductive consequence. This follows from $\mathrm{Tl}$ and the consideration that $\sim \mathrm{h}$ is logically equivalent to a disjunction of the $n-1$ remaining members of $P$. Thus, from T3 it follows that $p(m, b)$ is not greater than $p(\sim h, b)$. Thus, if $p(h, b)$ exceeds $p(\sim h, b)$, then there will be some member $m$ of $S$ such that $p(h, b)$ exceeds $p(m, b)$. This shows that if $p(h, b)$ exceeds $p(\sim h, b)$, then $I(h, b)$. If $p(h, b)$ does not exceed $p(\sim h, b)$, then it is not the case that $I(h, b)$ simply because the set containing just $h$ and $\sim h$ as members will be minimally inconsistent. This shows that $I(h, b)$ only if $p(h, b)$ exceeds $p(\sim h, b)$.

T6. If $d$ is a disjunction of two or more members of $P$, then $I(d, b)$ if and only if there is a $k$ which is a disjunct of $d$ such that $p(d, b)$ exceeds $p(\sim k, b)$. Consider any set $S$ of members of $G$ that includes a disjunction $d$ of two or more members of $P$ and is minimally inconsistent. For any disjunct $k$ of $d, S$ contains some member $m$ such $p(m, b)$ is not greater than $p(\sim k, b)$ by $T 4$. Hence if $k$ is a disjunct of $d$, and $p(d, b)$ exceeds $p(\sim k, b)$, there is a member $m$ of $S$ such that $p(d, b)$ exceeds $p(m, b)$. This shows that if $d$ is a disjunction of two or more members of $p$ and there is a $k$ which is a disjunct of $d$ such that $p(d, b)$ exceeds $p(\sim k, b)$, then $I(d, b)$. If there is no disjunct $k$ of $d$ such that $p(d, b)$ exceeds $p(\sim k, b)$, then the set of statements consisting of $d$ and the negations of each disjunct of $d$ will be a mini- 


\section{Keith Lehrer}

mally inconsistent set such that for no member $m$ of the set will it be the case that $p(d, b)$ exceeds $p(m, b)$. This shows that if $d$ is a disjunction of two or more members of $P$ and there is no $k$ which is a disjunct of $d$ such that $p(d, b)$ exceeds $p(\sim k, b)$, then it is not the case that $I(\mathrm{~d}, b)$.

T7. If $I(h, b)$ and $k$ is a deductive consequence of $h$, then $I(k, b)$. Proof: Let $h$ and $k$ be members of $G$ and such that $k$ is a deductive consequence of $h$ and $I(h, b)$. Either $h$ is a member of $P$ or it is not. Suppose $h$ is a member of $P$. In that case, either $k$ is logically equivalent to $h$ or $k$ is a disjunction of which $h$ is a disjunct. If $k$ is logically equivalent to $h$, then $I(k, b)$ by P2. If $k$ is a disjunction of which $h$ is a disjunct, $p(k, b)$ is at least as great as $p(h, b)$ by P4. But $p(h, b)$ is greater than $p(\sim h, b)$ by T5. Hence $p(k, b)$ is greater than $p(\sim h, b)$. Therefore, $I(k, b)$ by T6. This establishes the theorem on the supposition that $h$ is a member of $P$. Suppose $h$ is not a member of $P$. In this case, $h$ is a disjunction of members of $P$. Either $k$ is logically equivalent to $h$ or $k$ is a disjunction of members of $P$ having as disjuncts at least all those members of $P$ that are disjuncts of $h$. If $k$ is logically equivalent to $h$, then $I(k, b)$ by $P 2$. If $k$ has as disjuncts all those members of $P$ that are disjuncts of $h$, then $p(k, b)$ is at least as great as $p(h, b)$ by $P 4$. Since $I(h, b)$ by T6 it follows that there is some member $m$ of $P$ which is a disjunct of $h$ such that $p(h, b)$ is greater than $p(\sim m, b)$. But $m$ is also a disjunct of $k$. Thus $p(k, b)$ is also greater than $p(\sim m, b)$. Therefore $I(k, b)$ by $T 6$. This proves the theorem.

The next theorems concern the following two rules of acceptance:

AR. $A(h, b)$ if and only if either (i) $I(h, b)$ or (ii) there is some set of statements $S$ of which $h$ is a deductive consequence and such that any $k$ is a member of $S$ if and only if $I(k, b)$.

R. $A(h, b)$ if and only if either (i) $E(h, b)$ is strongly maximal or (ii) $h$ is a deductive consequence of the set $S$ which has as its only members $b$ and $k$ where $E(k, b)$ is strongly maximal.

T8. For any $s$ in $G, A(s, b)$ by AR if and only if either (a) $s$ is a member $m$ of $P$ such that for any other member $k$ of $P, p(m, b)$ exceeds $p(k, b)$ or $s$ is a deductive consequence of such a member $m$ and $b$, or (b) $s$ is a disjunction d of the members of a set $S$ of members of $P$ such that $m$ is a member of $S$ if and only if, for any member $k$ of $P$, if $p(m, b) \neq p(k, b)$, then $p(m, b)$ exceeds $p(k, b)$ or $s$ is a de- 
ductive consequence of such a disjunction $d$ and $b$. Proof: Either there is some member $m$ of $P$ such that for every other member $k$ of $P$, $p(m, b)$ exceeds $p(k, b)$ or there is not. Suppose there is such a member $\mathrm{m}$. In that case, by condition (i) of AR and $\mathrm{T} 6$ it follows that for every other member $k$ of $P, I(\sim k, b)$, and the set of such negations of other members of $P$ has $m$ as a deductive consequence. Hence by condition (ii) of $A R$, it follows that $A(m, b)$. Moreover, $I(b, b)$ from the fact that $p(b, b)=1$ by $P 3$, and thus there must be some $k$ in any minimally inconsistent set to which $b$ belongs such that $p(k, b)$ is less than 1. Hence by condition (i) of AR, $A(b, b)$. By condition (ii) of AR it follows that for any $s$ that is a deductive consequence of $m$ and $b$, $A(s, b)$. This proves that for any $s$ that satisfies condition (a) of the theorem $A(s, b)$. Suppose that there is no member $m$ of $P$ such that for any other member $k$ of $P, p(m, b)$ exceeds $p(k, b)$. In that case, there will be a disjunction $d$ of members of a set $S$ of members of $P$ such that $m$ is a member of $S$ if and only if for any member $k$ of $P$, if $p(m, b) \neq p(k, b)$, then $p(m, b)$ exceeds $p(k, b)$. For any member $k$ of $P$ that is not a disjunct of $k$ (if there are any), $I(\sim k, b)$ by T6, and the set of such negations of members of $P$ that are not disjuncts of $d$ has $d$ as a deductive consequence. (If the set is null, $d$ would be a disjunction of all members of $P$ and, hence, would be a deductive consequence of the set by natural deduction.) Hence $A(d, b)$ by condition (ii) of AR. Again, $A(b, b)$ by the argument given above. By condition (ii) of AR it follows that for any $s$ that is a deductive consequence of $d$ and $b, A(s, b)$. This proves that for any $s$ that satisfies condition (b) of the theorem $A(s, b)$ by AR. This shows that if $s$ satisfies either condition (a) or (b) of the theorem, then $A(s, b)$ by AR. We shall now prove that if $A(s, b)$ by $A R$, then $s$ satisfies either condition (i) or (ii) of the theorem. Again, either there is some member $m$ of $P$ such that, for every other member $k$ of $P, p(m, b)$ exceeds $p(k, b)$, or there is not. Suppose there is such a member. For any $s$ in $G$, either $s$ is a deductive consequence of $m$ and $b$ or it is not the case that $A(s, b)$ by AR. For consider the set $G^{\prime}$ of all those members of $G$ that are not deductive consequences of $m$ and $b$. Any $g$ that is a member of $G^{\prime}$ is such that the set whose only members are $m$ and $g$ is a minimally inconsistent set, because $m$ is inconsistent with every member of $G^{\prime}$ and no such member is itself inconsistent. But $p(m, b)$ exceeds $P(g, b)$ by $T 5, P 1$, and $P 4$. Hence for any such 


\section{Keith Lehrer}

$g$, it is not the case that $I(g, b)$ and thus it cannot be shown that $A(g, b)$ by condition (i) of AR. Thus, only those members $h$ of $G$ which are deductive consequences of $m$ and $b$ are such that $I(h, b)$, and no set of statements all of which are deductive consequences of $m$ and $b$ has any deductive consequence that is not a deductive consequence of $m$ and $b$ by the transitivity of deduction. This proves that $A(s, b)$ by AR only if $s$ satisfies condition (a) of the theorem on the supposition that there is some member $m$ of $P$ such that for any other member $k$ of $P, P(m, b)$ exceeds $p(k, b)$. Suppose now that there is no such member $m$. In that case, as we have seen, there is a disjunction $d$ of the members of a set $S$ of members of $P$ such that $\mathbf{m}$ is a member of $S$ if and only if, for any member $k$ of $P$, if $p(m, b) \neq p(k, b)$, then $p(m, b)$ exceeds $p(k, b)$. For any $s$ in $G$, either $s$ is a deductive consequence of $d$ and $b$ or it is not the case that $A(s, b)$ by AR. Assume $s$ is not a deductive consequence of $d$ and $b$. Then $P(s, b)$ is no greater than $1-f$ where for any $m$ that is a member of $S, p(m, b)=f$ by $P 1, P 3$, and P4. Thus, if $s$ is a member of $P$, then by the supposition it is not the case that $I(s, b)$ and, if $s$ is not a member of $P$, then it is a disjunction of members. Thus, if $s$ is a disjunction of members of $P$, then for any member $k$ that is a disjunct of $s, p(s, b)$ is not greater than $p(\sim \mathrm{k}, b)$ because no member $k$ of $P$ is such that $P(\sim k, b)$ is less than $f$. This follows from $\mathrm{P} 1, \mathrm{P} 3$, and $\mathrm{P} 4$. Hence it is not the case that $I(s, b)$ by T6. Moreover, if $s$ is not a disjunction of members of $P$, then it is a member of $P$, and, by supposition it is not the case that $I(s, b)$. Since a member $s$ of $G$ is either a disjunction of members of $P$ or a member of $P$, it follows that any $s$ that is a member of $G$ but not a deductive consequence of $d$ and $b$ is such that it is not the case that $I(s, b)$. Hence for any such $s$, it cannot be shown that $A(s, b)$ by condition (i) of AR. Thus, only those members $h$ of $G$ which are deductive consequences of $d$ and $b$ are such that $I(h, b)$, and no set of statements all of which are deductive consequences of $d$ and $b$ has any deductive consequence that is not a deductive consequence of $d$ and $b$. This proves that $A(s, b)$ by AR only if $s$ satisfies condition (b) of the theorem on the supposition that there is no member $m$ of $P$ such that for any other member $k$ of $P, p(m, b)$ exceeds $p(k, b)$. Since there either is or is not such a member, it follows that for any $s$ in $G, A(s, b)$ by AR only if $s$ satisfies either condition (a) or condition (b) of the theorem. 
T9. For any $s$ in $G, A(s, b)$ by $R$ if and only if either (a) $s$ is a member $m$ of $P$ such that for any other member $k$ of $P, P(m, b)$ exceeds $P(k, b)$ or $s$ is a deductive consequence of such a member $m$ and $b$, or (b) $s$ is a disjunction $d$ of the members of a set $S$ of all those members of $P$ such that $m$ is a member of $S$ if and only if, for any member $k$ of $P$, if $p(m, b) \neq p(k, b)$, then $p(m, b)$ exceeds $p(k, b)$ or $s$ is a deductive consequence of such a disjunction $d$ and $b$. Proof: By the definitions given in the text, for any member $m$ of $P$, there is a fixed number $q$ such that $E(m, b)=p(m, b)-q$. For any disjunction $d$ of a set of $\boldsymbol{n}$ different members mi of $P$,

$$
E(\mathrm{~d}, b)=\sum_{i=1}^{n} \mathrm{E}(\mathrm{mi}, b) / \mathrm{n} .
$$

It follows from these equalities by elementary algebra that if there is some member $m$ of $P$ such that for any other member $k$ of $P, p(m, b)$ exceeds $p(k, b)$, then $E(m, b)$ is strongly maximal. If there is no such member, then it follows that for some disjunction $d, E(d, b)$ is strongly maximal, where the members of $d$ belong to a set $S$ of members of $P$ such that $m$ is a member of $S$ if and only if, for any member $k$ of $P$, if $p(m, b) \neq p(k, b)$, then $p(m, b)$ exceeds $p(k, b)$. Therefore, $A(s, b)$ by $R$ if and only if $s$ satisfies either condition (a) or (b) of the theorem.

T10. $A(s, b)$ by AR if and only if $A(s, b)$ by $R$ for any $s$ in $G$. Proof: Immediate consequence of T8 and T9. 